\title{
Evaluating Architectural Design Schemes for Anganwadi Centre in Ajjarkad, Karnataka, India by Architectural Students Through Community Participatory Methods
}

International conference on Sustainable Development Research in the Asia-Pacific

SDRAP 2019: Sustainability in the Built Environment in the 21st Century: Lessons Learned from India and the Region pp 19-39 | Cite as

- Susan Ang (1) Email author (susan.ang@deakin.edu.au)

- Nandineni Ramadevi (2)

- Norwina Mohd Nawawi (3)

1. School of Architecture and Built Environment, Deakin University, , Geelong, Australia

2. Manipal School of Architecture and Planning, Manipal Academy of Higher Learning, , Manipal, India

3. Kulliyyah of Architecture and Environmental Design, International Islamic University Malaysia, , Selangor, Malaysia

Conference paper

First Online: 17 April 2021

- 18 Downloads

Part of the Environmental Science and Engineering book series (ESE)

\section{Abstract}

Anganwadi is a holistic health and education initiative that provides basic health care, nutrition and educational programs to rural mothers and children. It is part of the Indian Government's Integrated Child Development Services (ICDS) Scheme, introduced in 1975 and is today one of the largest child development initiatives in the world. The early developmental years of children aged o-6 years are spent in an Anganwadi or equivalent early learning centre that influences children's physical, emotional and intellectual development. A child not only engages in active learning but also engages in passive learning from its physical and environmental surroundings which are manifested in the architectural design of the building. A collaboration between architecture students from three universities in India, Australia and Malaysia as well as the office of the Deputy Director of Women and Child Welfare, Udupi District was initiated in 2018 for the proposed design of a purpose-built Anganwadi centre in Ajjarkad, Karnataka. The project was executed through a student global learning program known as iDiDe (Intercultural dialogue through design). This study evaluates the seven design schemes created with Ajjarkad community input and codesign participatory methods based upon sustainable integrated architectural 
parameters, including space efficiency, environmental resilience, children physical rating scale and cultural value. The issue of "fit for purpose" and "architecture design robustness" and subsequent construction of an Anganwadi centre are rarely interrogated at an integrated design level. In evaluation, this paper discusses the synthesis of architectural design, the pedagogy and cultural attributes that aid the formulation of architectural design parameters for Anganwadi building. The study found that through co-design participatory methods, the design process was shaped through community engagement and the outcomes thus satisfied the stakeholders as fit for purpose.

\section{Keywords}

Intercultural dialogue through design Anganwadi Participatory method This is a preview of subscription content, $\underline{\log } \underline{\text { in }}$ to check access.

\section{References}

Abdul Latip NS, Mohd Ariffin NA, Ang S (2013) Cultural Emersion 2013-Intercultural Dialogue Through Design. Kulliyyah of Architecture and Environmental Design, International Islamic University Malaysia (unpublished), Department of Architecture Google Scholar (http://scholar.google.com/scholar_lookup?

title=Cultural\%20Emersion\%202013-

Intercultural\%20Dialogue\%20Through\%2oDesign\&author=NS.\%20Abdul\%20Latip\& author=NA.\%20Mohd\%20Ariffin\&author=S.\%20Ang\&publication_year=2013)

Ali HH, Saba F, Nsairat Al (2009) Developing a green building assessment tool for developing countries-Case of Jordan. Build Environ 44(5):1053-1064

Google Scholar (https://scholar.google.com/scholar?

q=Ali\%20HH\%2C\%20Saba\%20F\%2C\%20Nsairat\%20Al\%20\%282009\%29\%2oDevel oping\%20a\%2ogreen\%2obuilding\%2oassessment\%20tool\%2ofor\%2odeveloping\%20 countries\%E2\%80\%93Case\%20of\%2OJordan.\%20Build\%20Environ\%2044\%285\%29 \%3А1053\%Е2\%80\%931064)

Ang S (2017a) Intercultural dialogue through design (iDiDe): a model of intercultural collaboration and student engagement. In: Tucker R (ed) Collaboration and student engagement in design education, vol 11. IGI Global, pp 230-256

Google Scholar (https://scholar.google.com/scholar? q=Ang\%20S\%20\%282017a\%29\%20Intercultural\%20dialogue\%20through\%2odesign \%20\%28iDiDe\%29\%3A\%20a\%20model\%20of\%2ointercultural\%2ocollaboration\%20 and\%20student\%20engagement.\%20In\%3A\%20Tucker\%20R\%20\%28ed\%29\%20Coll aboration\%20and\%20student\%2oengagement\%2oin\%2odesign\%2oeducation\%2C\%2 ovol\%2011.\%20IGI\%20Global\%2C\%20pp\%20230\%E2\%80\%93256)

Ang S (2017b) iDiDe Overview. Issue date: 5 November 2017 SRR 367 and SRR 767 Built Environment Study Tour 2017, Deakin University Unit Guides (published as part of course work content)

Google Scholar (https://scholar.google.com/scholar?

q=Ang\%20S\%20\%282017b\%29\%2oiDiDe\%20Overview.\%20Issue\%2odate\%3A\%205 \%20November\%202017\%20SRR\%20367\%20and\%20SRR\%20767\%20Built\%20Envir onment\%20Study\%20Tour\%202017\%2C\%20Deakin\%2oUniversity\%2oUnit\%20Guid es\%20\%28published\%20as\%20part\%20of\%20course\%20work\%20content\%29)

Ang S (2018) Collaboration and Dialogue, Exhibition Catalogue, Intercultural Dialogue through Design, Anganwadi, Ajjarkad, Udupi, Manipal University Press, India 
Google Scholar (https://scholar.google.com/scholar?

q=Ang\%20S\%20\%282018\%29\%20Collaboration\%20and\%20Dialogue\%2C\%2oExhibi tion\%20Catalogue\%2C\%2OIntercultural\%2oDialogue\%2othrough\%2oDesign\%2C\%2 oAnganwadi\%2C\%20Ajjarkad\%2C\%20Udupi\%2C\%2oManipal\%2oUniversity\%2oPre ss\%2C\%2oIndia)

Anthony K (2002) Designing for diversity: implications for architectural education in the twenty-first century. J Archit Educ 55(4):257-267.

https://doi.org/10.1162/104648802753657969

(https://doi.org/10.1162/104648802753657969)

CrossRef (https://doi.org/10.1162/104648802753657969)

Google Scholar (http://scholar.google.com/scholar_lookup?

title=Designing\%2ofor\%20diversity\%3A\%20implications\%2ofor\%20architectural\%2 oeducation\%20in\%20the\%20twenty-

first\%20century\&author=K.\%20Anthony\&journal=J\%20Archit\%20Educ\&volume=55 \&issue $=4$ \&pages $=257-$

267\&publication_year $=2002 \& d o i=10.1162 \% 2 F 104648802753657969)$

Berardi U (2013) Clarifying the new interpretations of the concept of sustainable building. Sustain Cities Soc 8:72-78

CrossRef (https://doi.org/10.1016/j.scs.2013.01.008)

Google Scholar (http://scholar.google.com/scholar_lookup?

title=Clarifying\%20the\%20new\%20interpretations\%20of\%2othe\%20concept\%20of\% 20sustainable\%2obuilding\&author=U.\%20Berardi\&journal=Sustain\%20Cities\%20So $c \&$ volume $=8 \&$ pages $=72-78 \&$ publication_year $=2013$ )

Broome J (2005) Should we value population? J Polit Philos 13(4):399-413

CrossRef (https://doi.org/10.1111/j.1467-9760.2005.00230.x)

Google Scholar (http://scholar.google.com/scholar_lookup?

title=Should\%20we\%2Ovalue\%2opopulation\%3F\&author=J.\%20Broome\&journal=J \%20Polit\%20Philos\&volume=13\&issue=4\&pages=399-413\&publication_year=2005）

Brundtland Report (1987) Report of the World Commission on environment and development. Our common future. UN Documents: gathering a body of Global Agreements has been compiled by the NGO committee on education of the conference of NGOs from United Nations web sites with the invaluable help of information and communications technology

Google Scholar (https://scholar.google.com/scholar?

q=Brundtland\%20Report\%20\%281987\%29\%20Report\%20of\%20the\%20World\%20C ommission\%20on\%2oenvironment\%20and\%2odevelopment.\%20Our\%20common\% 2ofuture.\%2oUN\%2oDocuments\%3A\%20gathering\%20a\%2obody\%20of\%2oGlobal\% 20Agreements\%2ohas\%2obeen\%20compiled\%20by\%20the\%20NGO\%20committee \%20on\%20education\%20of\%2othe\%20conference\%20of\%20NGOs\%2ofrom\%2oUnit ed\%2oNations\%20web\%20sites\%20with\%2othe\%2oinvaluable\%2ohelp\%20of\%2oinf ormation\%20and\%20communications\%2otechnology)

Chiu ML (2002) An organizational view of design communication in design collaboration. Des Stud 23(2):187-210

CrossRef (https://doi.org/10.1016/S0142-694X(01)ooo19-9)

Google Scholar (http://scholar.google.com/scholar_lookup?

title=An\%20organizational\%20view\%20of\%2odesign\%20communication\%2oin\%2od esign\%20collaboration\&author=ML.\%20Chiu\&journal=Des\%20Stud\&volume=23\&iss ue $=2 \&$ pages $=187-210 \&$ publication_year $=2002$ )

India OGDP (2018) Anganwadi Centres, Government of India. https://data.gov.in/dataset-group-name/anganwadi-centers 
(https://data.gov.in/dataset-group-name/anganwadi-centers). Retrieved o2 June 2018

Integrated Child Development Services, MoWaCD, Government of India, (2009)

Integrated Child Development Services (ICDS) Scheme, Ministry of Women and Child Development. https://www.icds-wcd.nic.in/icds.aspx (https://www.icdswcd.nic.in/icds.aspx). Retrieved 29 June, 2018

Jacobus A, Du Pisani (2006) Sustainable development-historical roots of the concept. Environl Sci 3(2):83-96

Google Scholar (https://scholar.google.com/scholar?

q=Jacobus\%20A\%2C\%20Du\%20Pisani\%20\%282006\%29\%20Sustainable\%2odevelo pment\%E2\%80\%93historical\%20roots\%20of\%20the\%20concept.\%20Environl\%20Sc i\%203\%282\%29\%3A83\%E2\%80\%9396)

Lawson L (2005) Dialogue through design: the east St. Louis neighbourhood design workshop and south end neighbourhood plan. Landscape J 24(2):157-171

Google Scholar (https://scholar.google.com/scholar?

q=Lawson\%20L\%20\%282005\%29\%20Dialogue\%2othrough\%2odesign\%3A\%2othe\% 20east\%20St.\%20Louis\%2oneighbourhood\%2odesign\%2oworkshop\%20and\%20sout h\%20end\%2oneighbourhood\%2oplan.\%2oLandscape\%20J\%2024\%282\%29\%3A157 $\% \mathrm{E} 2 \% 80 \% 93171)$

Mohd Nawawi N (2018) Cultural interpretations in design, exhibition catalogue, intercultural dialogue through design. Manipal University Press, Anganwadi, Ajjarkad, Udupi, India

Google Scholar (http://scholar.google.com/scholar_lookup?

title=Cultural\%2ointerpretations\%2oin\%2odesign\%2C\%20exhibition\%2ocatalogue\% 2C\%20intercultural\%20dialogue\%2othrough\%2odesign\&author=N.\%2oMohd\%20Na wawi\&publication_year=2018)

Nandineni R (2018) Contribution to social architecture, exhibition catalogue, intercultural dialogue through design. Manipal University Press, Anganwadi, Ajjarkad, Udupi, India

Google Scholar (http://scholar.google.com/scholar_lookup?

title=Contribution\%20to\%20social\%20architecture\%2C\%20exhibition\%20catalogue \%2C\%2ointercultural\%20dialogue\%20through\%20design\&author=R.\%20Nandineni \&publication_year=2018)

Orpett Long S, Akande YS, Purdy RW, Nakano K (2010) Deepening learning and inspiring rigor: Bridging academic and experiential learning using a host country approach to study tour. J Stud Int Educ 14(1):89-111

CrossRef (https://doi.org/10.1177/1028315308327952)

Google Scholar (http://scholar.google.com/scholar_lookup?

title=Deepening\%2olearning\%20and\%2oinspiring\%2origor\%3A\%2oBridging\%20aca demic\%20and\%2oexperiential\%2olearning\%2ousing\%20a\%2ohost\%2ocountry\%20a pproach\%20to\%20study\%20tour\&author=S.\%20Orpett\%2OLong\&author=YS.\%2OAk ande\&author=RW.\%20Purdy\&author=K.\%20Nakano\&journal=J\%20Stud\%20Int\%20 Educ\&volume=14\&issue =1\&pages $=89-111 \&$ publication_year=2010)

Palliyaguru R, Karunasena G, Ang S (2018) Review on sustainable building design and construction in the rural context: the case of building Ampara, Sri Lanka. Sustainable development research in the Asia-Pacific Region. Springer, Cham, pp. 493-507 Google Scholar (https://scholar.google.com/scholar?

q=Palliyaguru\%20R\%2C\%20Karunasena\%20G\%2C\%20Ang\%20S\%20\%282018\%29\% 20Review\%20on\%20sustainable\%2obuilding\%2odesign\%20and\%20construction\%20 in\%2othe\%2orural\%2ocontext\%3A\%2othe\%20case\%20of\%2obuilding\%20Ampara\% 2C\%2oSri\%2oLanka.\%20Sustainable\%2odevelopment\%2oresearch\%2oin\%2othe\%2 
oAsia-

Pacific\%20Region.\%20Springer\%2C\%20Cham\%2C\%20pp.\%20493\%E2\%80\%93507)

Poggenpohl SH (2008) Practicing collaborative action in design in Sharon Helmer

Poggenpohl, Sanoff H, "Multiple views of participatory design" in Archnet-IJAR. Int J

Archit Res 2(1):57-69. Retrieved at https://www.archnet-

ijar.net/index.php/IJAR/article/viewFile/177/241 (https://www.archnet-

ijar.net/index.php/IJAR/article/viewFile/177/241)

Sanoff H (2006) Origin of community design. Planner's Network.

https://www.plannersnetwork.org/2006/o1/origins-of-community-design/

(https://www.plannersnetwork.org/2006/o1/origins-of-community-design/).

Retrieved 2 Jan 2006

Sato K (Ed) (2009) Design Integrations: Research and Collaboration. Intellect Books

Google Scholar (https://scholar.google.com/scholar?

q=Sato\%20K\%20\%28Ed\%29\%20\%282009\%29\%20Design\%20Integrations\%3A\%20

Research\%20and\%2oCollaboration.\%20Intellect\%2oBooks)

The Anganwadi Project (2013) Our Approach, The Anganwadi Project Incorporated. https://www.anganwadiproject.com/projects/approach/

(https://www.anganwadiproject.com/projects/approach/). Retrieved o4 July 2018

Winschiers-Theophilus H, Bidwell NJ, Blake E (2012) Community consensus: design beyond participation. Des Issues 28(3):89-100

CrossRef (https://doi.org/10.1162/DESI_a_o0164)

Google Scholar (http://scholar.google.com/scholar_lookup?

title=Community\%20consensus\%3A\%2odesign\%2obeyond\%2oparticipation\&author $=$ H.\%20Winschiers-

Theophilus\&author=NJ.\%20Bidwell\&author=E.\%2oBlake\&journal=Des\%2oIssues\&v olume $=28 \&$ issue $=3 \&$ pages $=89-100 \&$ publication $\_$year $=2012$ )

\section{Copyright information}

(C) The Author(s), under exclusive license to Springer Nature Switzerland AG 2021

\section{About this paper}

Cite this paper as:

Ang S., Ramadevi N., Nawawi N.M. (2021) Evaluating Architectural Design Schemes for Anganwadi Centre in Ajjarkad, Karnataka, India by Architectural Students Through Community Participatory Methods. In: Iyer-Raniga U. (eds) Sustainability in the Built Environment in the 21st Century: Lessons Learned from India and the Region. SDRAP 2019. Environmental Science and Engineering. Springer, Cham.

https://doi.org/10.1007/978-3-030-61891-9_2

- First Online 17 April 2021

- DOI https://doi.org/10.1007/978-3-030-61891-9_2

- Publisher Name Springer, Cham

- Print ISBN 978-3-030-61890-2

- Online ISBN 978-3-030-61891-9

- eBook Packages Education Education (으).

- Buy this book on publisher's site

- Reprints and Permissions 


\section{Personalised recommendations}

\section{SPRINGER NATURE}

(C) 2020 Springer Nature Switzerland AG. Part of $\underline{\text { Springer Nature. }}$

Not logged in Not affiliated 175.137.231.148 